\title{
Breakthrough, Concern and Prospect: Research on the Communication of Tiktok Rural Short Video from the Perspective of Scene Theory
}

\author{
Fang Xuelan \\ School of Humanities and Communication, Zhejiang Gongshang University, Hangzhou, China
}

Email address:

Jau1998@163.com

\section{To cite this article:}

Fang Xuelan. Breakthrough, Concern and Prospect: Research on the Communication of Tiktok Rural Short Video from the Perspective of Scene Theory. Science Innovation. Vol. 9, No. 4, 2021, pp. 124-128. doi: 10.11648/j.si.20210904.11

Received: April 15, 2021; Accepted: May 17, 2021; Published: May 24, 2021

\begin{abstract}
With the development of Internet technology and the popularization of mobile intelligent terminals, mobile short video has developed rapidly in recent years. In particular, short video platforms represented by Tiktok and Kuaishou have penetrated into People's Daily life, while short video platforms such as Tiktok and Kuaishou have gradually become important carriers for People's Daily sharing and social communication. In the era of mobile Internet, scene, as a new entrance, is reshaping the intelligent connection between platform, users and diversified content services. The five elements of scene have become the core driving force, prompting us to enter a scene development era soon. As a major branch of Tiktok short videos, rural short videos construct scenes of rural life, display diversified features of rural scenes through immersive experience and sensory interactive experience, arouse people's memories of the countryside, win the audience's affection, and realize the spread of rural culture in the way of scene. Therefore, based on the scene theory, this paper analyzes the scene construction of Tiktok rural short videos and studies its value. By reflecting on the problems in the development of Tiktok rural short videos, it provides constructive thinking for its development and has certain significance for the future construction of rural short videos.
\end{abstract}

Keywords: Scene Theory, Tiktok Village Short Video, Internet, Social Media

\section{突破·忧思·展望：场景理论视阈下抖音乡村短视频的传播研究}

\section{方雪蓝}

浙江工商大学人文与传播学院, 杭州, 中国

\section{邮箱}

Jau1998@163.com

摘要：随着互联网技术的发展和移动智能终端的普及，移动短视频近年来发展如火如茶，尤其是以抖音和快手为代表 的短视频平台已经深入了人们的日常生活, 抖音和快手等短视频平台也逐渐成为人们日常分享和社会交往的重要载体。 而在移动互联网时代，场景作为一个新的入口正在重塑着平台、用户和多样化的内容服务之间的智能化连接，场景的 五要素成为核心驱动力，促使我们即将进入一个场景化的发展时代。作为抖音短视频一大分支的乡村类短视频构建了 乡村生活场景, 通过沉浸式的体验和感官上的交互体验展示了多样化的乡村场景特征, 唤醒人们对于乡村的记忆, 赢 得受众的喜爱, 实现了乡村文化的场景化传播。因此，本文将基于场景理论对于抖音乡村类短视频的场景构建进行分 析以及其价值进行研究，通过对抖音乡村短视频发展中出现的问题进行反思，为其发展提供建设性思考，对乡村类短 视频的未来建设有一定的意义。 
关键词: 场景理论, 抖音乡村短视频, 互联网, 社交媒体

\section{1. 引言}

随着互联网技术的普及和移动短视频的爆发式增长, 我国短视频行业正迎来发展的红利期, 从移动短视频行业 的兴起、增长到爆发, 短视频也在深刻改变着人们获取信 息的重要方式，满足了碎片化时代人们的阅读娱乐需求。 2014年我国迎来了短视频发展元年, 近两年我国短视频行 业也是呈现着井喷式爆发增长, 据第47次《中国互联网络 发展状况统计报告》显示, 我国网络视频用户规模达到了 9.27亿, 占网民整体的 $93.7 \%$, [1]短视频的内容形式多种 多样, 移动短视频构建的场景丰富了受众的体验。作为移 动短视频发展领域佼佼者的抖音短视频更是成为用户自 我表达和交流互动的重要平台, 抖音短视频聚集了来自世 界各地不同领域的创作者, 创作了丰富多彩的内容。尤其 是近年来关于乡村短视频的内容创作吸引了众多粉丝关 注, 抖音乡村类短视频通过展示或者构建乡村生活场景, 勾起了用户对乡村生活的回忆, 在场景化时代, 场景作为 入口构建的乡村短视频既是在为全国各地的网民提供了 自我表达的舞台和视觉展示空间, 也为我国乡村发展赋能, 让移动短视频带动乡村经济发展。目前学界基于场景理论 研究短视频还是比较少的, 搜索知网主要有: 杨飞霞的 《场景理论视域下短视频原生广告传播研究》是基于场景 理论对短视频的原生广告进行分析 [2]; 姚梦科的《媒介场 景理论视域下短视频的发展——以抖音为例》则是借助场 景理论对抖音短视频的场景建构进行研究并分析其对用 户行为产生的影响 [3]; 申艾的《场景理论视域下PGC短视 频的场景建构研究》以场景理论为切入点, 以 $\mathrm{PGC}$ 短视频作 为研究对象,探析PGC短视频的场景建构 [4]; 傅慧的《场 景理论视角下移动社交短视频的价值研究》也是从场景理 论出发,对移动社交短视频进行场景构建的研究分析, 并反 思在此过程中会出现的问题[5]。从场景理论出发研究乡村 内容传播的论文主要有: 郭婉君和于春生的《场景理论视 域中乡村直播的价值与忧思》对乡村直播的场景构建进行 分析并指出存在的问题[6]; 黎玲的《乡村文旅融合对游客 满意度的影响研究——基于场景理论的实证分析》借助场 景理论,构建乡村文旅融合下的旅游满意度模型, 探讨了
乡村文旅融合对游客满意度的影响[7]。基于场景理论研究 乡村短视频传播的相关学术论文更是非常少, 主要有李晶 晶的《技术视角下土味文化短视频的虚拟与现实》从技术 视角出发研究 “土味短视频” 传播过程中出现的各种问题 [8], 陈斯亮的《快手短视频中的乡村景象呈现研究》研究 快手短视频中所展现的乡村景象,分析农村短视频用户群 体的日常生活的媒介展现[9]。因此本研究从场景理论视角 切入, 以抖音为例研究乡村短视频的场景构建具有一定的 研究价值。

\section{2. 场景理论}

\section{1. 场景理论溯源及其应用发展}

场景理论最初的理论溯源是戈夫曼的 “拟剧理论” ， [10]戈夫曼认为个人在前台和后台的形象是不一样的, 在 社会的大舞台上人们塑造着自己的形象, 在后台人们会摘 下表演的面具, 展现的是最真实的自己, 人们在前台和后 台展示的形象有明显的不同。后来学者梅罗维茨在戈夫曼 的“拟剧理论”和麦克卢汉的媒介理论的基础上提出了 “场 景理论” , 他认为媒介营造的环境会给人们的思想和行为 都带来巨大的影响。[11]尤其是在现代社会互联网技术的 发展、 $5 \mathrm{G}$ 时代的到来和电子媒介逐渐普及, 会放大这种影 响。罗伯特・斯考伯和谢尔・伊斯雷尔两位学者在2014年出 版的书籍《即将到来的场景时代》中提出了关于场景的“五 力” , 即移动设备、社交媒体、传感器、大数据和定位系 统, 这些场景因素深刻影响着人们的日常生活, 决定着人 们对媒介情境的感知。[12]

我国学者彭兰教授认为 “移动时代场景的意义大大强 化, 移动传播的本质是基于场景的服务, 即对场景 (情境) 的感知即信息 (服务) 适配。” [13]场景在媒介传播的空 间氛围和虚拟情景营造中扮演着重要角色, 尤其是在移动 社交媒体时代, 是基于场景为受众提供的服务。河南理工 大学的郜书锴教授也提到场景理论中的五力将会成为当 前互联网和信息技术发展的重要驱动力, 在未来五力会更 多地运用到媒体融合发展和信息生产中去。[14]

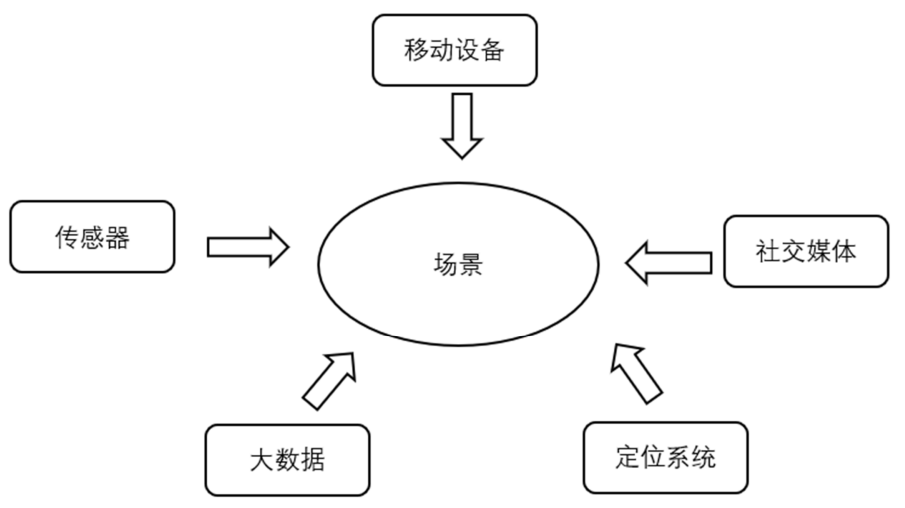

图1 场景“五力”意图。 


\section{2. 移动短视频中的场景构建}

移动短视频作为一种新的信息生产传递和生活娱乐 的重要方式已经融入到受众的日常生活, 营造的丰富场景 给受众带来了多样的场景体验。移动短视频作为一个集社 交、自我展示和信息分享一体的平台, 搭建的生活化场景 让身处于不同场景下的用户有着相似的场景体验。在构建 场景中包含着硬要素和软要素, 硬要素主要是场所和景别, 场景可以分为“场”和“景”两个部分，场所既包括了视频中 展现的场所也包括了观看者所处的场所, 景别包含了短视 频中的景和受众获取的景。[12]软要素即空间氛围和社交 氛围, 移动短视频充分利用了互联网时代人们碎片化的阅 读特征, 通过营造虚拟场景和真实场景, 让短视频中的各 种要素和用户之间智能连接。

在社交媒体时代, 移动短视频在渗透用户日常生活的 同时也填充了人们的碎片化时间, 人们可以利用乘交通工 具、排队、吃饭休息等时间刷短视频获取新闻信息和娱乐。 移动短视频通过设置评论、点赞、转发、打赏等功能, 营 造了一个虚拟化的社交圈层, 构建移动短视频的互动社交 场景。移动短视频通过多重场景构建不仅满足了用户的消 遣娱乐需求, 还获得了不同场景的体验感知, 使用户在观 看短视频中获得了满足感。

\section{3. 抖音乡村短视频中的场景构建}

\section{1. 移动设备与传感器打破时空限制}

以手机、电脑和平板为代表的移动智能终端便利了人 们连接互联网获取信息, 这类移动设备已经成为了我们身 体的一部分, 它们使得媒介载体在任何时间和地点都能成 为人们的延伸。随着时代发展, 数字脱贫地开展, 使得乡 村地区也普及了互联网技术和移动电子设备。人们可以利 用移动智能设备记录下生活场景或者趣事, 然后通过简单 的剪辑加入特效并配上音乐上传至抖音平台, 现实场景和 虚拟场景相结合完成作品创作。近年来, 更多的人通过移 动智能终端加入到了抖音乡村短视频的创作中来, 既有以
李子染为代表的专业创作团队, 也有像 “农村玉米妹妹” 这样的草根创作团队。这类短视频通过展现乡村生活场景, 或者再加入一些当下的流行元素, 获得了受众的青睐。

对于用户而言, 通过移动设备看抖音短视频也更加便 利, 无论处于什么时间和空间都可以第一时间获取最新的 最热门的短视频。同时传感器等技术的应用能够更加精确 捕捉用户信息, 这类信息也能更好地帮助平台和创作者进 行内容生产。正如喻国明教授所认为的, 传感器获取信息 能够突破时空维度, 不再仅限于人们的触感听觉和视觉, 可以获得更多维度的信息。[16]同时平台在内容生产过程 中还可以对收集的内容信息进行处理检测, 更够让内容生 产更加有效有质。

\section{2. 大数据与定位系统提供精准定位与场景适配}

抖音乡村短视频最大的特点是具有浓厚的乡村气息, 无论展示的是乡村美食、乡村生活或者是对乡村文化行为 的模仿都无法脱离浓浓的乡土气息。随着农村网民数量的 增长, 扩大了这类短视频的创作大军, 同时现在很多人经 历了城市快节奏的生活, 习惯了各种高楼大厦, 乡村短视 频获得了越来越多人的喜爱, 这类短视频的创作可以唤起 他们对乡村的记忆。根据抖音每年发布的大数据都可以显 示关于乡村短视频创作者在不断增加, 内容题材也越来越 丰富。

场景的构建是为提供特定情境之下的适配服务, 因此 大数据和定位系统发挥着核心作用, 能够帮助内容更加精 准触达用户。同时大数据和定位系统还可以获得关于用户 观看喜好、地理位置、社交图谱等重要的信息, 借助这类 信息可以给用户推送他们喜爱的乡村短视频。[17]例如每 逢春节大家都会返乡过年或者去到乡村拜访亲戚, 现在很 多人也喜欢返璞归真, 选择到乡村地区旅游, 这时就可以 通过定位系统为受众推荐附近乡村类短视频, 处于乡村场 景之下观看乡村短视频会有更强的临场感。大数据技术可 以记录用户的观看数据, 并对这一系列数据进行分析, 根 据用户的喜好创作推送他们喜欢观看的抖音乡村短视频。

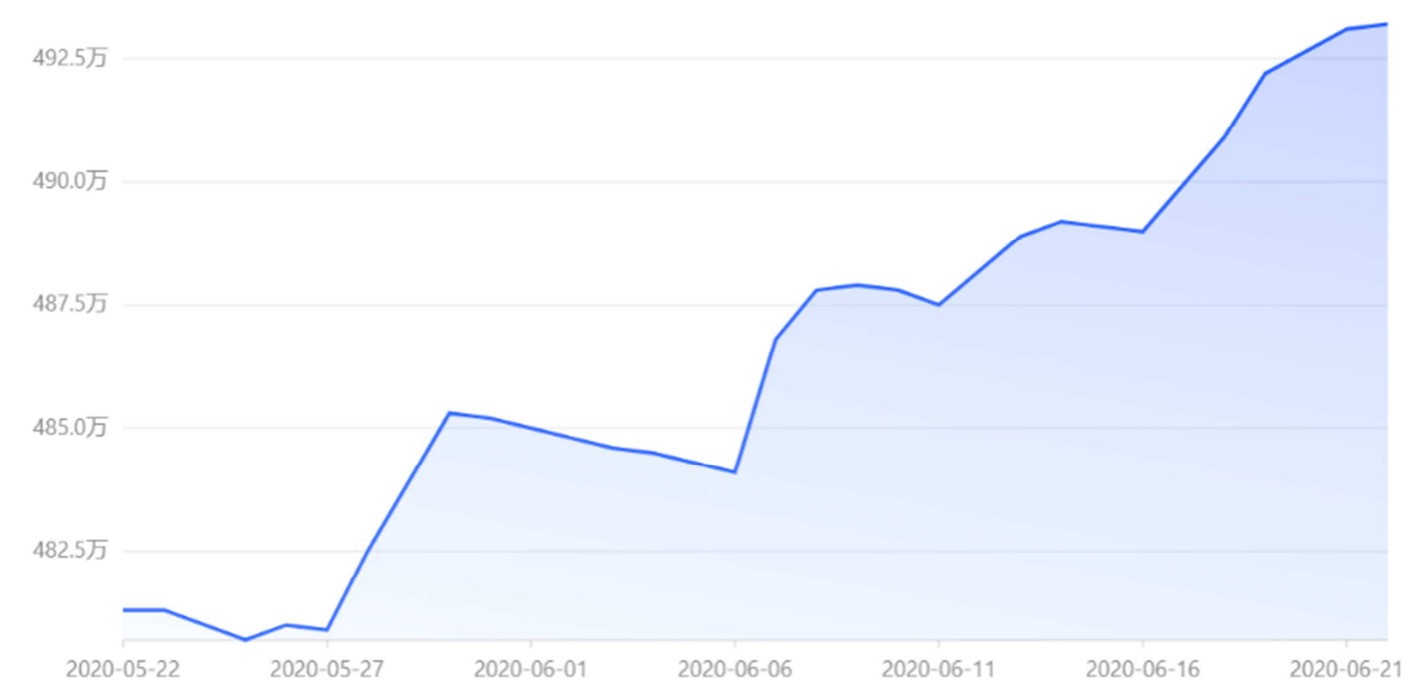

图2 抖音乡村短视频创作者“晓凡凡”2020年5-6月粉丝趋势。 


\section{3. 社交媒体实现情感传播与双向互动}

抖音乡村短视频借助社交媒体构建的乡村生活场景, 可以实现和用户之间的情感传播, 从而产生对乡村生活的 情感共鸣, 激发人们的乡村记忆。有些短视频通过拍摄原 汁原味的乡村生活图景, 展示了最原始的乡村生活, 种菜、 做饭、养殖等场景。例如李子染就是通过记录乡村生活, 拍摄农作物种植生长, 做饭等场景, 再经过后期的剪辑处 理形成原创性的抖音乡村短视频。而抖音短视频创作者 “晓凡凡” 则是以 “一个人模仿全村人” 的表演形式, 展 现了“早上的农村”、“农村看电影”、“农村收废品” 等一系列乡村短视频, 营造的场景氛围迎合了受众需求, 获得了百万点赞, 其中表 1 是对创作者 “晓凡凡” 抖音短 视频的点赞数进行的梳理。“晓凡凡” 在2020年5到6月粉 丝数量就增长了十几万, 受众纷纷表示从他创作的乡村短 视频中找寻到了关于乡村的回忆, 创作者通过乡村短视频 中构建的生活场景和用户之间实现了情感传播。

表1 抖音乡村短视频创作者“晓凡凡”乡村系列短视频点赞量（截至2021 年4月14日）。

\begin{tabular}{ll}
\hline 短视频主题 & 点赞数 \\
\hline 早上的农村 & 216.4 万 \\
回家后碰到村里办酒席时 & 152.2 万 \\
回家后村里邻居来你家串门时 & 117.4 万 \\
农村小时候的回忆 & 114.7 万 \\
农村炸爆米花 & 131.9 万 \\
那些年和妈妈在地里干活 & 100.4 万 \\
\hline
\end{tabular}

在社交媒体时代，用户可以借助短视频平台进行社交 和双向互动。用户身处不同情景之下仍可以看到创作者展 示构建的乡村场景。在抖音短视频中还可以通过点赞、评 论、打赏等功能和创作者或者其他网民进行交流互动,虚 拟的互动场景迎合了用户的社交需求。抖音乡村短视频的 创作者还可以根据受众的互动反馈生产出更加符合受众 需求的更高质量的短视频, 增强用户黏性, 从而提高抖音 平台乡村短视频的创作质量。

\section{4. 抖音乡村短视频的问题反思和未来展望}

尽管抖音乡村短视频构建的真实场景和虚拟场景迎 合了众多受众的需求, 有着广阔的发展前景, 同时还有更 多的创作者源源不断地加入抖音乡村短视频的内容生产 中来, 但是抖音乡村短视频在发展过程中还存在着一些不 足。首先，抖音乡村短视频的创作者还是以乡村地区的民 众为主, 他们可以第一手记录下乡村生活景象, 但是有很 多民众的媒介素养还是不够的, 创作出的内容以及构建的 场景可能存在着违法违规的风险。其次, 抖音乡村短视频 构建的场景是虚拟场景和现实场景的结合, 但是二者并没 有存在着明显的界限, 很可能会影响用户的价值判断。最 后, 抖音乡村短视频的发展能够带动乡村旅游和乡村产业 的发展, 但是也容易受到资本的影响, 使得视频创作单单 为了追逐利益, 遭到用户反感, 违背了抖音乡村短视频的 创作初衷。
因此, 创作者对于乡村短视频的场景构建要满足以下 要求。第一, 抖音乡村短视频的创作者要具备一定的社会 责任以及法律和道德意识, 积极构建真实美好的乡村生活 场景, 传播正能量, 创作出更多更高质量的乡村短视频, 同时还要学习一定互联网络法律法规, 避免生产出低俗违 规的内容。第二, 用户是短视频创作的第一生产力, 抖音 乡村短视频创作者还要及时和受众互动交流, 获得他们的 意见反馈和观看感受, 为乡村短视频创作注入更多动力源 泉; 第三, 乡村短视频在一定程度可以成为带动乡村经济 发展的重要支柱, 创作者要构建有价值有内涵的乡村生活 情景, 让乡村短视频成为带动乡村人民发展的致富之路。

\section{5. 结语}

随着智媒时代的到来，社交媒体兴起，每个人都可以 加入到移动短视频创作中去, 为移动短视频的发展注入了 新动力。大数据、传感器、定位系统等新兴技术会被更多 的应用到短视频的内容生产和数据分析中去, 不断开拓内 容创作的新领域, 迎合现代消费社会中受众喜好。但是随 着移动短视频平台地不断发展, 短视频行业的竞争会不断 加大, 因此如何抓住用户需求和喜好会成为短视频发展中 越来越重视的议题, 构建符合大众化需求的场景是移动短 视频获得更多流量的关键。[15]在即将到来的场景时代, 社交化、场景化、个性化是移动短视频创作的核心要素, 与此同时还要注重和受众之间的深层次连接, 挖掘更多的 场景式社交体验。

在移动短视频发展大军里, 抖音短视频异军突起, 而 抖音乡村短视频已经成为抖音短视频内容创作中的重要 部分。抖音乡村短视频具有乡土性、原创性、社交性、趣 味性等特征, 同时借助场景五力, 构建了虚拟场景和真实 场景, 向受众展示了乡村生活图景, 了解到了中国的乡土 社会。乡村短视频构建的场景有助于唤起乡村记忆, 传承 乡村文化, 若能积极解决发展中的困难问题, 定会有广阔 的发展前景。但是互联网时代各种各样的场景正在发生着 变化, 移动短视频的创作也容易偏离创作初衰, 因此, 在 构建移动短视频场景的过程中不能脱离核心要义和社会 发展的价值导向。

\section{参考文献}

[1] 第 47 次《中国互联网络发展状况统计报告》 [EB/OL].[2021-0203].http://cnnic.cn/gywm/xwzx/rdxw/20172 017_7084/202102/t20210203_71364.htm

[2] 杨飞霞. 场景理论视域下短视频原生广告传播研究[D].江 西财经大学,2019.

[3] 姚梦科.媒介场景理论视域下短视频的发展一一以抖音为 例 [J].新闻研究导刊,2019,10(13):234-235.

[4] 申艾. 场景理论视域下PGC短视频的场景建构研究[D].江 西师范大学,2019. 
[5] 傅慧.场景理论视角下移动社交短视频的价值研究 [J].东南 传播,2018(09):30-32.

[6] 郭婉君,于春生.场景理论视域中乡村直播的价值与忧思 [J]. 电视研究,2019(12):38-40.

[7] 黎玲.乡村文旅融合对游客满意度的影响研究——基于场 景理 论的实证分析 [J]. 技术经济与管理研 究,2021(04):100-104.

[8] 李晶晶.技术视角下土味文化短视频的虚拟与现实[J].声屏 世界,2021(01):105-106.

[9] 陈斯亮. 快手短视频中的乡村景象呈现研究 $[D]$. 华中师范 大学,2019.

[10] 欧文・戈夫曼.日常生活的自我呈现 $[\mathrm{M}]$. 冯钢, 译. 北京大学 出版社, 2008:93, 203-204.

[11] (美) 约书亚・梅罗维茨.消失的地域[M]. 肖志军译. 北京:清 华大学出版社, 2002:2, 16, 31 .
[12] 罗伯特・斯考伯，谢尔・伊斯雷尔.即将到来的场景时代 $[\mathrm{M}]$. 赵乾坤，周宝曜，译.北京: 北京联合出版公司, 2014:11, 71.

[13] 彭兰. 场景: 移动时代媒体的新要素 [J]. 新闻 记 者,2015(03):20-27.

[14] 郜书锴. 场景理论: 开启移动传播的新思维 [J]. 新闻 界,2015(17):44-48+58.

[15] 赵程.场景理论下的 “火山小视频” 的场景建构 [J].东南传 播,2018(03):119-120.

[16] 喻国明.内容科技:未来传媒的全新生产力[J]. 教育传媒研 究,2020(03):6-8.

[17] 王军峰. 场景化思维: 重建场景、用户与服务连接 $[\mathrm{J}]$. 新 闻与写作，2017,(2):97-99.

[18] 马宁. 移动互联网络的场景构建与传播模式变迁 $[\mathrm{J}]$. 现 代传播, 2016(6):138-142. 